$\underline{\text { Preprint typeset in JHEP style. - HYPER VERSION }}$

\title{
P-Vortices, Gauge Copies, and Lattice Size
}

\author{
Roman Bertle \\ Inst. für Kernphysik, Technische Universität Wien, A-1040 Vienna, Austria \\ E-mail: bertle@kph.tuwien.ac.at \\ Manfried Faber \\ Inst. für Kernphysik, Technische Universität Wien, A-1040 Vienna, Austria \\ E-mail: faber@kph.tuwien.ac.at \\ Jeff Greensite \\ The Niels Bohr Institute, Blegdamsvej 17, DK-2100 Copenhagen Ø, Denmark \\ E-mail: greensit@alf.nbi.dk

\section{Štefan Olejník} \\ Institute of Physics, Slovak Academy of Sciences, SK-842 28 Bratislava, Slovakia \\ E-mail: fyziolej@savba.sk
}

\begin{abstract}
We study the dependence of the center-projected string tension on both the lattice size, and the number of gauge copies used for maximal center gauge fixing. We show that a recent finding of Bornyakov, Komarov, Polikarpov, and Veselov (hep-lat/0002017), indicating a substantial breakdown of center dominance in maximal center gauge, is only obtained for rather small lattice volumes, and is not found in numerical simulations on larger lattices. It is shown that center-projected Creutz ratios approach the full asymptotic string tension as lattice size increases, and that the P-vortex density is consistent, at moderately weak couplings, with 2-loop scaling behavior.
\end{abstract}

Keywords: Confinement, Lattice Gauge Field Theories, Solitons Monopoles and Instantons. 


\section{Contents}

1. Introduction 1

2. $N_{\text {copy }}$ and Lattice Size Dependence 3

2.1 Gauge-Fixing Convergence Criterion 5

3. Vortex Density 6

4. Vortex Thickness 9

5. Conclusions

\section{Introduction}

In the last few years there has been renewed interest, within the lattice gauge theory community, in the center vortex theory of confinement [1]. The revival of this old idea is due to a number of numerical studies, which all indicate that center vortices are ubiquitous in the QCD vacuum and give rise to the linear confining potential [2-15]. Recently, however, a paper by Bornyakov, Komarov, Polikarpov, and Veselov (BKPV) [16] appeared which questions the validity of a procedure, known as center projection in maximal center gauge, which was essential in many of these studies. The claim is that when large numbers of random gauge copies are used in fixing the gauge (in an effort to minimize the Gribov copy problem), the center-projected string tension underestimates the full string tension by as much as 30\%. Our purpose in the present article is to show that, while BKPV have certainly raised an important issue, their actual data was strongly affected by the rather small lattice volumes used in the numerical simulations. We will show that the conclusions drawn from this data are not sustained by simulations on larger lattices, which generally agree with our previously reported results [4].

In the direct version of maximal center gauge [4], the procedure is to maximize

$$
R=\sum_{\mu} \sum_{x} \operatorname{Tr}_{A}\left[U_{\mu}(x)\right]
$$

by an iterative over-relaxation procedure, where $\operatorname{Tr}_{A}[U]$ is the trace of $U$ in the adjoint representation. Let $R_{n}$ denote the value of $R$ after $n$ over-relaxation sweeps. When $R_{n}$ is judged to have converged, e.g. according to a criterion of the form

$$
\frac{R_{n}-R_{n-50}}{R_{n}}<\delta
$$


then the link variables $U_{\mu}(x)$ are projected onto the nearest center element $Z$. In $\mathrm{SU}(2)$ lattice gauge theory, the projection is simply

$$
Z_{\mu}(x)=\operatorname{sign} \operatorname{Tr}\left[U_{\mu}(x)\right]
$$

Center-projected Wilson loops, Creutz ratios, etc. are observables computed from the center-projected link variables. It was shown in a number of studies (see, in particular, ref. [4]), that

- thin vortex excitations of the projected lattice, known as "P-vortices", are located roughly in the middle of thick center vortices on the unprojected lattice;

- projected Creutz ratios $\chi_{c p}(I, I)$ are close to the asymptotic string tension on the unprojected lattice ("center dominance");

- the density of P-vortices, from $\beta=2.3$ onward, scales according to asymptotic freedom;

- removing center vortices (located via the projected lattice) from unprojected lattices also removes confinement and chiral symmetry breaking, and brings the topological charge on the lattice to zero [6].

Because these numerical results have such strong implications for the QCD confinement mechanism, it is important that the center projection procedure be examined critically. The first obvious question - why should this procedure work at all? - was addressed in ref. [17]. There it was shown that in the absence of Gribov copies (i.e. if the gauge can be fixed to a global maximum of $R$ ), then center projection in maximal center gauge will always locate a thin vortex inserted anywhere on the lattice. This was dubbed the "vortex-finding property" of maximal center gauge, and is certainly a necessary condition for its success. However, the vortices found in the QCD vacuum are not thin vortices, but are necessarily of finite thickness in physical units. Moreover, maximal center gauge is plagued with Gribov copies, since the over-relaxation scheme converges only to a local maximum of $R$, which will be slightly different for every gauge copy of a given lattice configuration.

In view of the Gribov copy problem, and the finite thickness of vortices, one must rely on empirical checks of vortex-finding via center projection. In this article we will study the sensitivity of center-projected Creutz ratios and the P-vortex density with respect to: (i) the number $N_{\text {copy }}$ of random gauge copies used for maximal center gauge fixing; (ii) the lattice size; and (iii) the convergence parameter $\delta$ in eq. (1.2). We will here only discuss center projection in direct maximal center gauge, since this is the case treated in ref. [16], and it is also the center gauge with which we have the most experience. It should be noted, however, that alternatives to maximal center gauge do exist; these include Laplacian center gauge [7] (which is free of the Gribov copy problem), as well as two other recent proposals $[18,19]$. 


\section{2. $N_{c o p y}$ and Lattice Size Dependence}

We begin with the $N_{\text {copy }}$ dependence, whose importance was recently emphasized by BKPV [16]. As noted above, when the over-relaxation gauge-fixing procedure is applied to different gauge copies of a given lattice configuration, slightly different values of $R$ are obtained. One way to minimize the gauge copy dependence is to carry out the over-relaxation procedure on a number $N_{\text {copy }}$ of random gauge copies, perform center projection on the copy with the largest value of $R$, and evaluate observables. Data obtained in this way, over a range of $N_{\text {copy }}$ values, can then be extrapolated to the $N_{\text {copy }} \rightarrow \infty$ limit. $^{1}$ In the original simulations of ref. [4], only three gauge copies were used, and there was no attempt to extrapolate to $N_{\text {copy }} \rightarrow \infty$. Projected Creutz ratios $\chi_{c p}(I, I)$ were found to be close to the asymptotic string tensions reported by Bali et al. [21], for all $I \geq 2$. BKPV, however, calculate Creutz ratios in the range $N_{\text {copy }} \in[1,20]$, and extrapolate to $N_{\text {copy }} \rightarrow \infty$ by fitting their data to the functional form

$$
\chi_{c p}^{N_{c o p y}}(I, I)=\chi_{c p}(I, I)+\frac{c(I, I)}{\sqrt{N_{c o p y}}}
$$

The result reported by BKPV is that projected string tensions, at $\beta=2.4,2.5$, underestimate the full string tension by about $20 \%$ at $N_{\text {copy }}=20$, and by as much as $30 \%$ in the extrapolation to $N_{\text {copy }} \rightarrow \infty$. This result suggests that the center dominance previously reported in maximal center gauge was a numerical accident, a result of using too few gauge copies.

However, apart from the number of gauge copies, there is one other notable difference between the BKPV simulations and previous work. This is the matter of lattice size. Data reported in ref. [4] was obtained at $\beta=2.3$ and $\beta=2.4$ on $16^{4}$ lattices, and at $\beta=2.5$ on a $22^{4}$ lattice. BKPV, on the other hand, used only $12^{4}$ lattices at $\beta=2.3$ and $\beta=2.4$, and lattice size $16^{4}$ at $\beta=2.5$. This raises the question of whether the BKPV results, obtained on the smaller lattices, were seriously contaminated by finite-size effects. To find out, we have repeated the center-projection calculation at $\beta=2.3$ and $\beta=2.5$ on a variety of lattice sizes, for $N_{\text {copy }} \in[1,20]$. For the convergence parameter in eq. (1.2), we have used $\delta=2 \times 10^{-7}$.

In Fig. 11 we display results for the Creutz ratio $\chi_{c p}^{N_{c o p y}}(4,4)$ vs. $N_{\text {copy }}$ at $\beta=2.5$, for lattice sizes ranging from $8^{4}$ to $28^{4}$. Two features of this data are immediately apparent. First, there is indeed a slow downward trend in the Creutz ratio as $N_{\text {copy }}$

\footnotetext{
${ }^{1} \mathrm{P}$-vortex positions extracted from different Gribov copies are closely correlated, as one would expect if they locate physical objects [4]. It is possible, however, by choosing a very special starting configuration with links gauge-fixed to have mainly positive trace, to destroy the vortex-finding property of center projection even for thin vortices [17,20]. This is one aspect of the Gribov copy problem in maximal center gauge. Since the vortex-finding property is essential to center projection, it is evident that any unusual variation of the iterative gauge-fixing procedure, which destroys this property, must be avoided.
} 


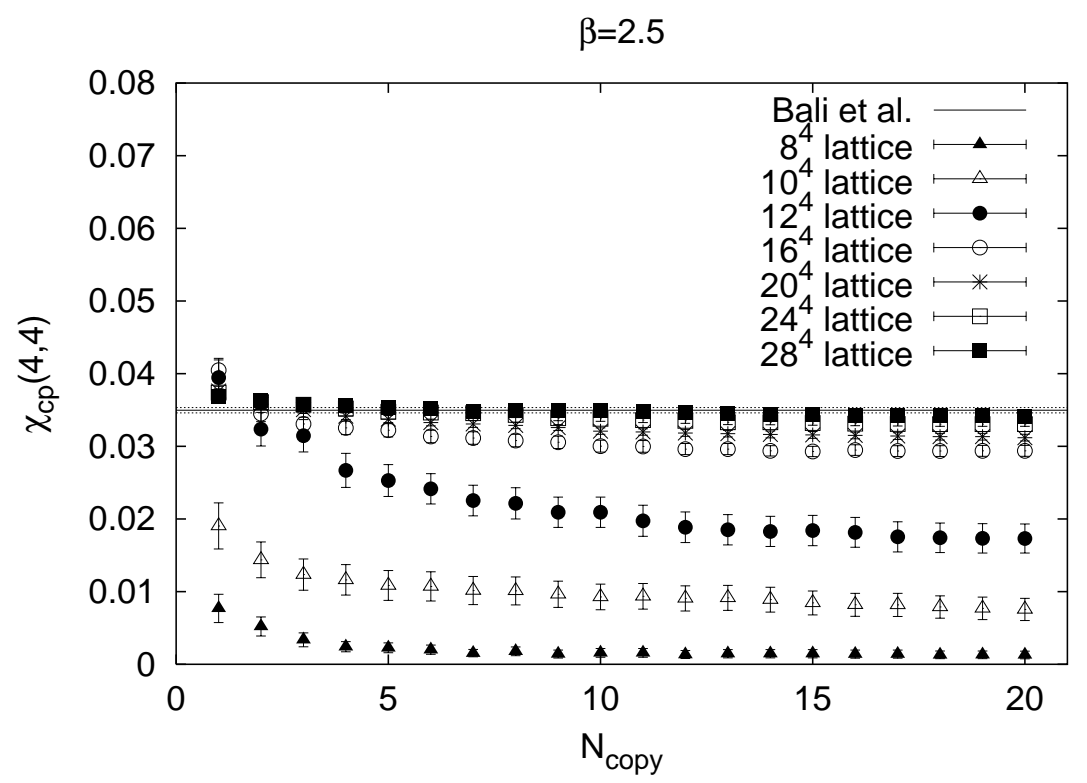

Figure 1: Center projected Creutz ratios $\chi_{c p}^{N_{c o p y}}(4,4)$ vs. $N_{c o p y}$ at various lattice volumes, for $\beta=2.5$. The solid line in this figure, and in Figs. 246, indicates the asymptotic string tension extracted by standard methods on unprojected lattices, reported by Bali et al. [21]. Dashed lines indicate the errorbar in this asymptotic string tension.

increases, as noted by BKPV, but this effect is much more pronounced on smaller lattices than on larger lattices. Second, although Creutz ratios on the smaller lattices grossly underestimate the full string tension, the data appears to steadily increase towards the full asymptotic string tension, reported by Bali et al. [21], as the lattice size increases. These trends in the data are by no means unique to the particular Creutz ratio $\chi_{c p}(4,4)$ at $\beta=2.5$, but are typical of all of our results. For completeness we display, in Figs. 2 and 3 , some other projected Creutz ratios $\chi_{c p}^{N_{\text {copy }}}(I, I)$ for $I$ in the range $I=2-5$, at couplings $\beta=2.3$ and $\beta=2.5$. In Figs. 2-6, solid (dashed) lines indicate the value (errorbar) of the asymptotic string tension on the unprojected lattice, reported in ref. [21].

In Fig. 4 we show the projected Creutz ratios $\chi_{c p}^{N_{c o p y}}(I, I)$ for $N_{c o p y}=5,10,15,20$ on the largest lattices we have used: $20^{4}$ at $\beta=2.3$ and $\beta=2.4$, and $28^{4}$ at $\beta=2.5$. We also show the values of these Creutz ratios extrapolated to the $N_{\text {copy }} \rightarrow \infty$ limit, using the fitting function (2.1) suggested by BKPV. As usual in maximal center gauge, all the $\chi_{c p}(I, I)$ for $I \geq 2$ are close to the asymptotic string tension, and these latest results are not far from our earlier results reported in ref. [4]. As another way of showing lattice size dependence, we take the average of the projected $\left(N_{\text {copy }} \rightarrow \infty\right)$ 

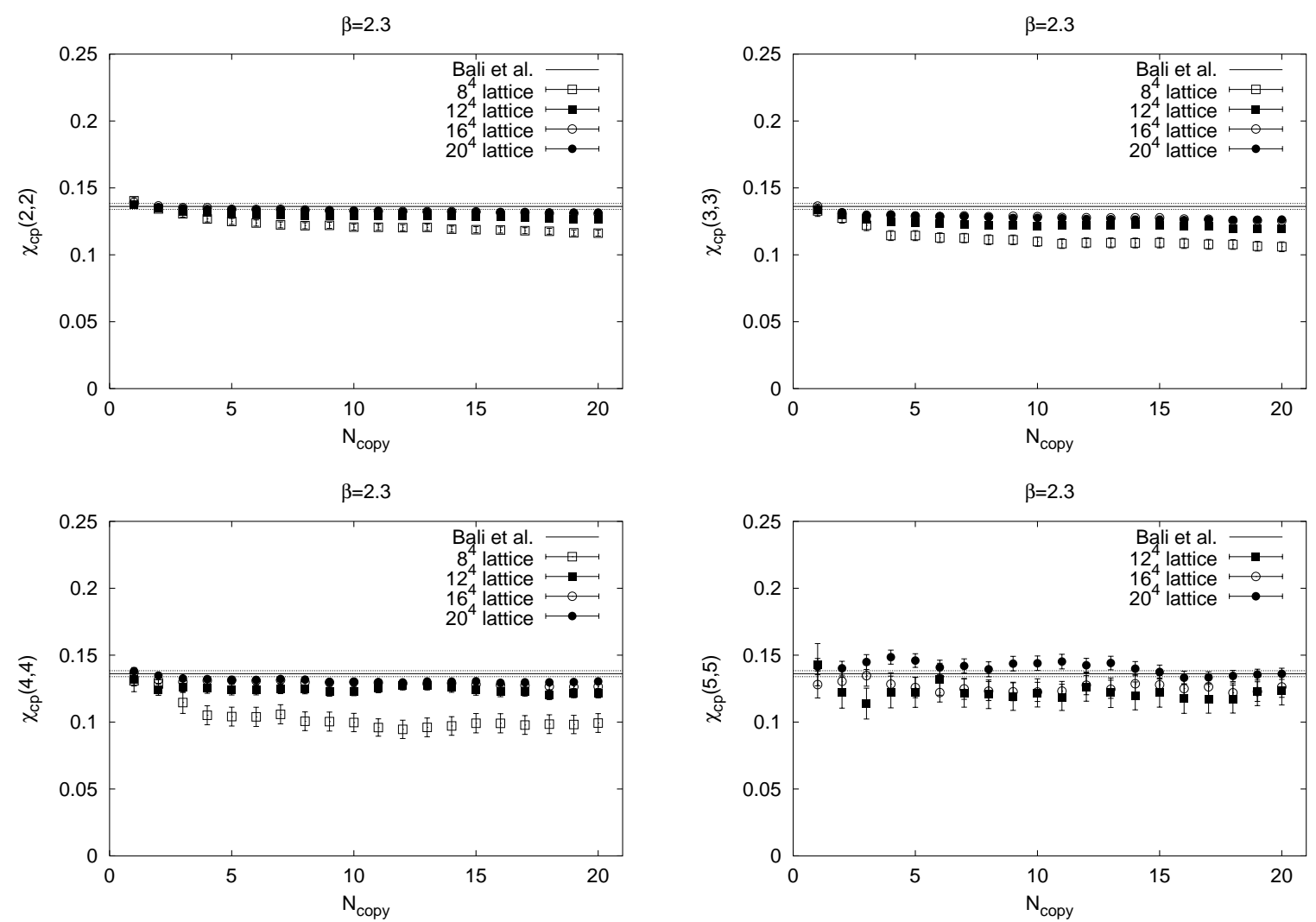

Figure 2: Center projected Creutz ratios $\chi_{c p}^{N_{c o p y}}(I, I)$ vs. $N_{c o p y}$ and lattice volume, at $\beta=2.3$ and $I=2-5$.

Creutz ratios $\chi_{c p}(I, I)$ in the range $I=2-5$

$$
\chi_{a v} \equiv \frac{1}{4} \sum_{I=2}^{5} \chi_{c p}(I, I)
$$

Figure 5 shows the lattice size dependence of $\chi_{a v}$. Note the approach of $\chi_{a v}$ to the full asymptotic string tension $\left(\sigma_{S U(2)}=0.035\right.$ at $\left.\beta=2.5\right)$ as lattice size increases.

\subsection{Gauge-Fixing Convergence Criterion}

In addition to lattice volume and $N_{\text {copy }}$ dependence, it is also worthwhile to check that the numerical results are stable when the gauge-fixing convergence criterion is strengthened, i.e. when the constant $\delta$ in eq. (1.2) is reduced. When $\delta$ is chosen too large, the center projected Creutz ratios come out significantly too high. This is illustrated in Fig. 6, which shows results for projected Creutz ratios with convergence criteria $\delta=10^{-2}, 10^{-3}, 10^{-4}, 2 \times 10^{-7}$ at $\beta=2.5$ (24 lattice). The weakest convergence criterion, corresponding to $\delta=10^{-2}$, is clearly insufficient for accurate results, but Creutz ratios obtained with the two smallest values of $\delta$ are fairly consistent, indicating that these numbers are not far from the $\delta \rightarrow 0$ limit. 

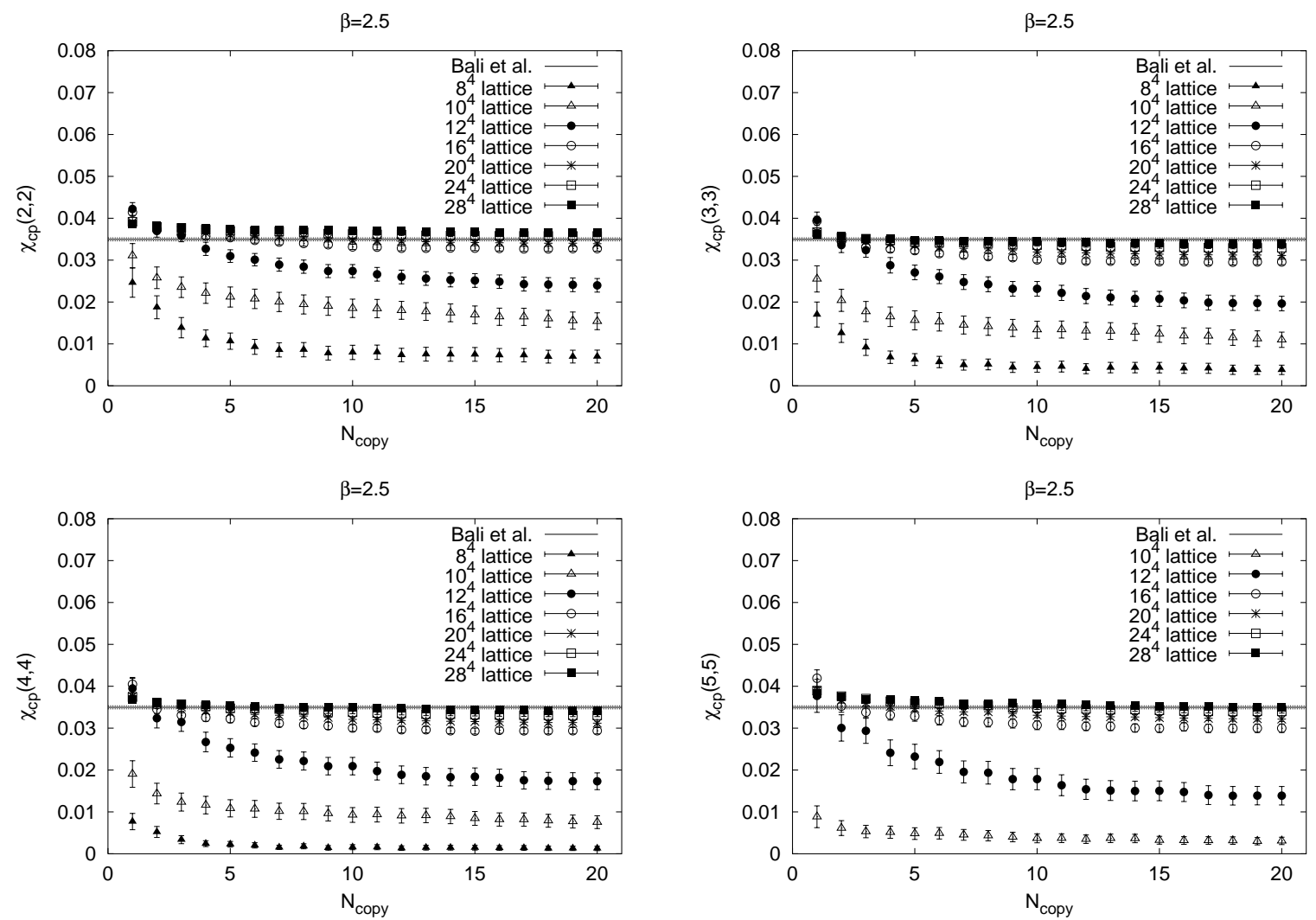

Figure 3: Center projected Creutz ratios $\chi_{c p}^{N_{c o p y}}(I, I)$ vs. $N_{c o p y}$ and lattice volume, at $\beta=2.5$ and $I=2-5$.

\section{Vortex Density}

Next we consider the scaling of the vortex density. The lattice P-vortex density $p$ is the total number of $\mathrm{P}$-vortex plaquettes (i.e. plaquettes on the projected lattice with $Z Z Z Z=-1$ ), divided by the total number of plaquettes on the lattice. This quantity is proportional to the average area taken up by $\mathrm{P}$-vortices per unit lattice volume, and is determined from the center-projected plaquette expectation value

$$
p=\frac{1}{2}\left(1-W_{c p}[1,1]\right)
$$

If this quantity scales as predicted by asymptotic freedom, then we would have $p=p_{a f}$, where

$$
p_{a f}=\frac{\rho}{6 \Lambda^{2}} F(\beta)
$$

Here $\rho$ is the vortex density (average vortex area per unit volume) in physical units of inverse area, and

$$
F(\beta)=\left(\frac{6 \pi^{2}}{11} \beta\right)^{102 / 121} \exp \left[-\frac{6 \pi^{2}}{11} \beta\right]
$$




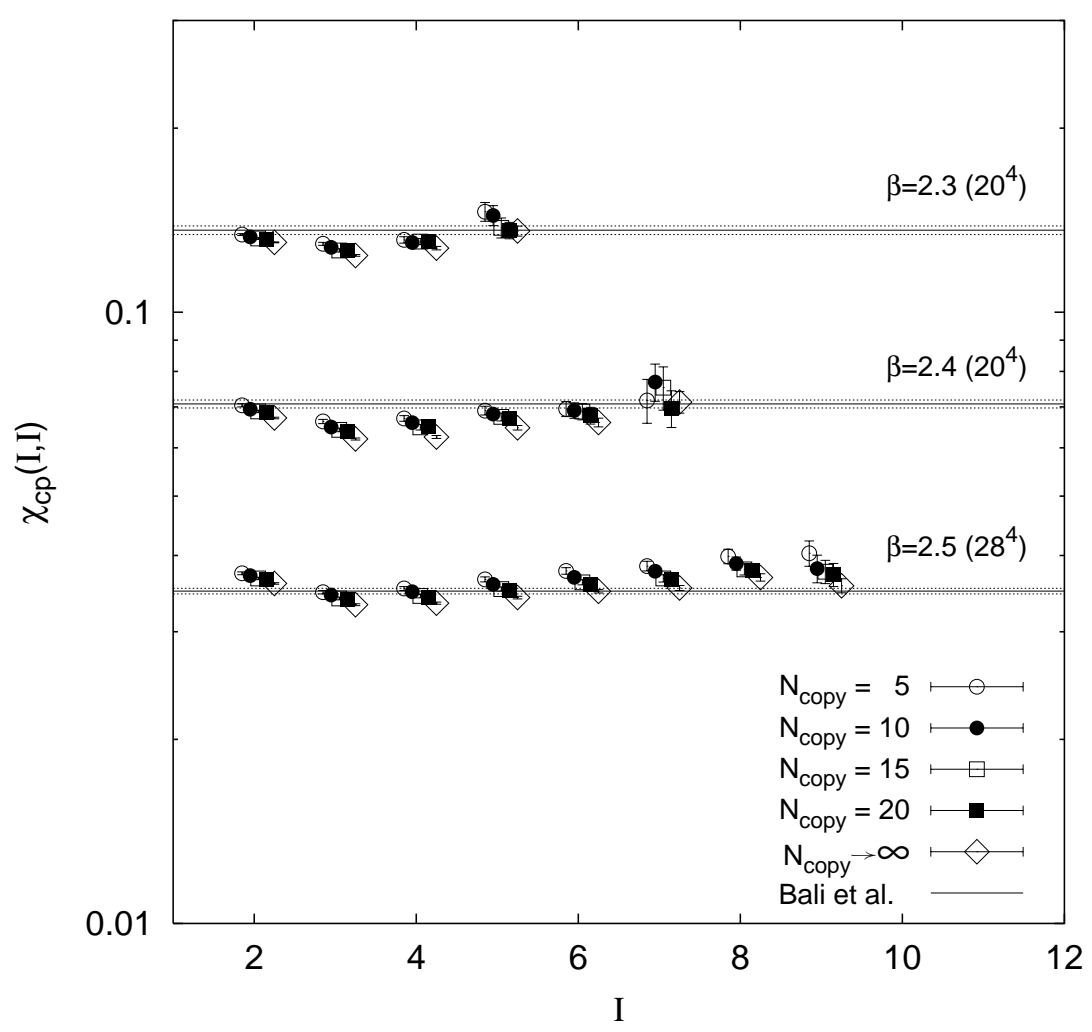

Figure 4: Center-projected Creutz ratios $\chi_{c p}(I, I)$ at $N_{c o p y}=5,10,15,20$, and extrapolated to $N_{\text {copy }} \rightarrow \infty$, on the largest lattices $\left(20^{4}\right.$ lattices at $\beta=2.3,2.4$, and $28^{4}$ at $\left.\beta=2.5\right)$ used in our simulations.

In Fig. 7 we plot the lattice vortex density rescaled by the asymptotic freedom expression

$$
\tilde{p} \equiv \frac{p}{F(\beta)}
$$

which should be constant in the large $\beta$ limit, if $p$ scales according to asymptotic freedom. There appears to be good evidence for this kind of scaling, already for $\beta \geq 2.2$, in agreement with previous results $[4,10]$. In Fig. 1 and Table 1, the vortex densities at $\beta=2.3,2.4,2.5$ are taken from the largest lattices $\left(20^{4}, 20^{4}\right.$ and $28^{4}$, respectively), and extrapolated to the $N_{\text {copy }} \rightarrow \infty$ limit by a fit to

$$
p^{N_{c o p y}}=p+\frac{c}{\sqrt{N_{\text {copy }}}}
$$

The vortex densities at other values of $\beta$ (with $N_{\text {copy }}=3$ ) are just taken from our previous work. For comparison, in Table [, we display our values for $\tilde{p}$, and the values of the rescaled asymptotic string tension

$$
\tilde{\sigma} \equiv \sigma_{S U(2)} / F(\beta)
$$




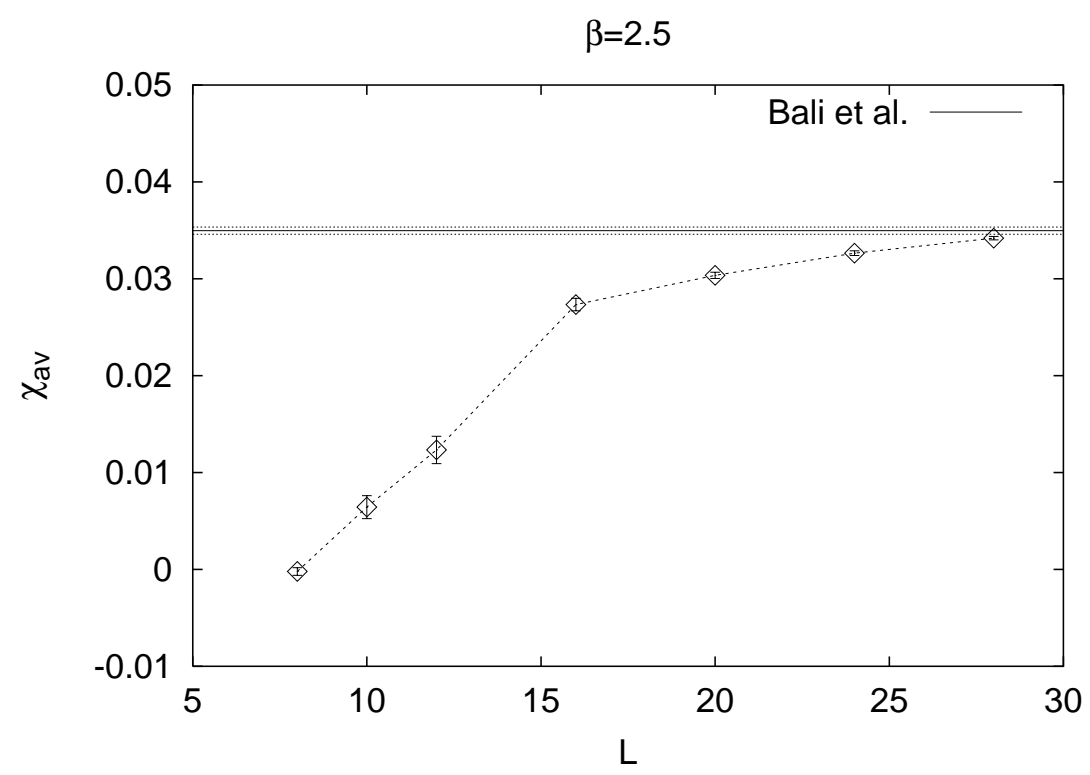

Figure 5: Average of projected, $N_{c o p y} \rightarrow \infty$ extrapolated Creutz ratios $\chi_{c p}(I, I)$, in the range $I=2-5$.

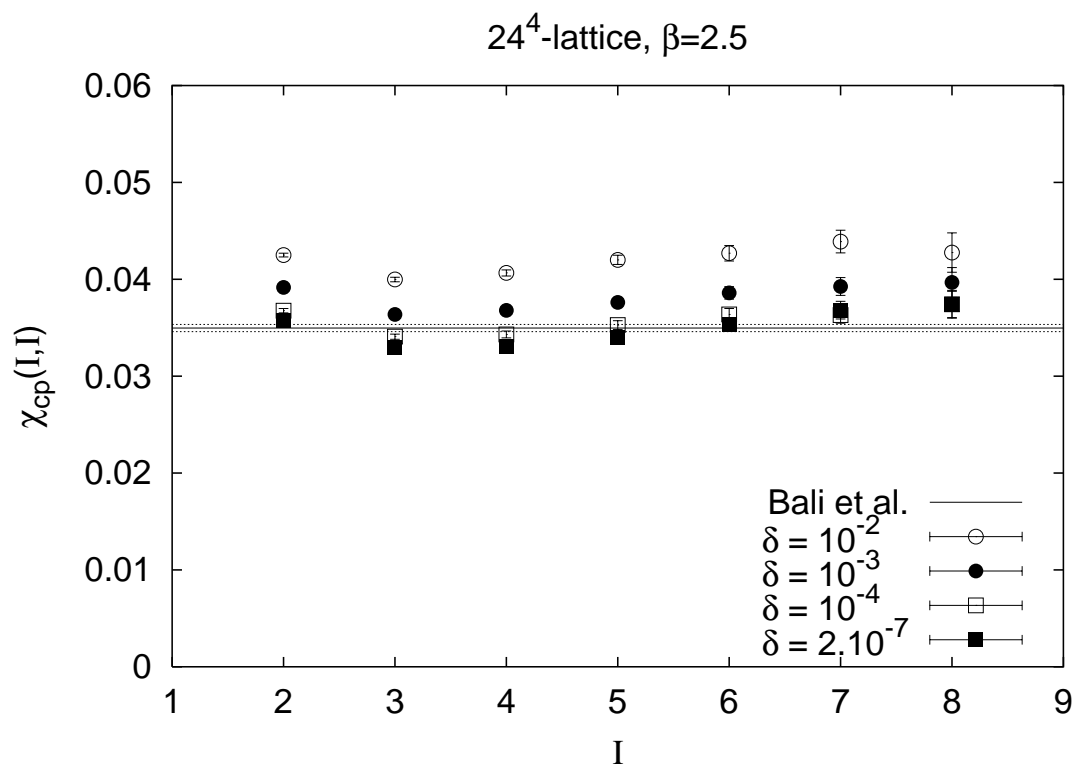

Figure 6: Effect of varying the convergence criterion $\delta$ on projected Creutz ratios (extrapolated to $N_{\text {copy }} \rightarrow \infty$ ) at $\beta=2.5$ on a $24^{4}$ lattice.

where $\sigma_{S U(2)}$ is the string tension (in lattice units) on the unprojected lattice, at the given $\beta$ value. It is interesting to note that the scaling of vortex density $p$, in the range $\beta=2.3-2.5$, is substantially better than the the scaling of the full asymptotic 


\begin{tabular}{|c|c|c|}
\hline$\beta$ & $\tilde{p}$ & $\tilde{\sigma}$ \\
\hline 2.3 & $2.51(2) \times 10^{3}$ & $3.89(6) \times 10^{3}$ \\
2.4 & $2.47(3) \times 10^{3}$ & $3.34(5) \times 10^{3}$ \\
2.5 & $2.35(3) \times 10^{3}$ & $2.73(3) \times 10^{3}$ \\
\hline
\end{tabular}

Table 1: Rescaled vortex density $\tilde{p}$ and $\mathrm{SU}(2)$ string tension $\tilde{\sigma}$. These quantities should be constant in the scaling limit.

string tension $\sigma_{S U(2)}$ in this range.

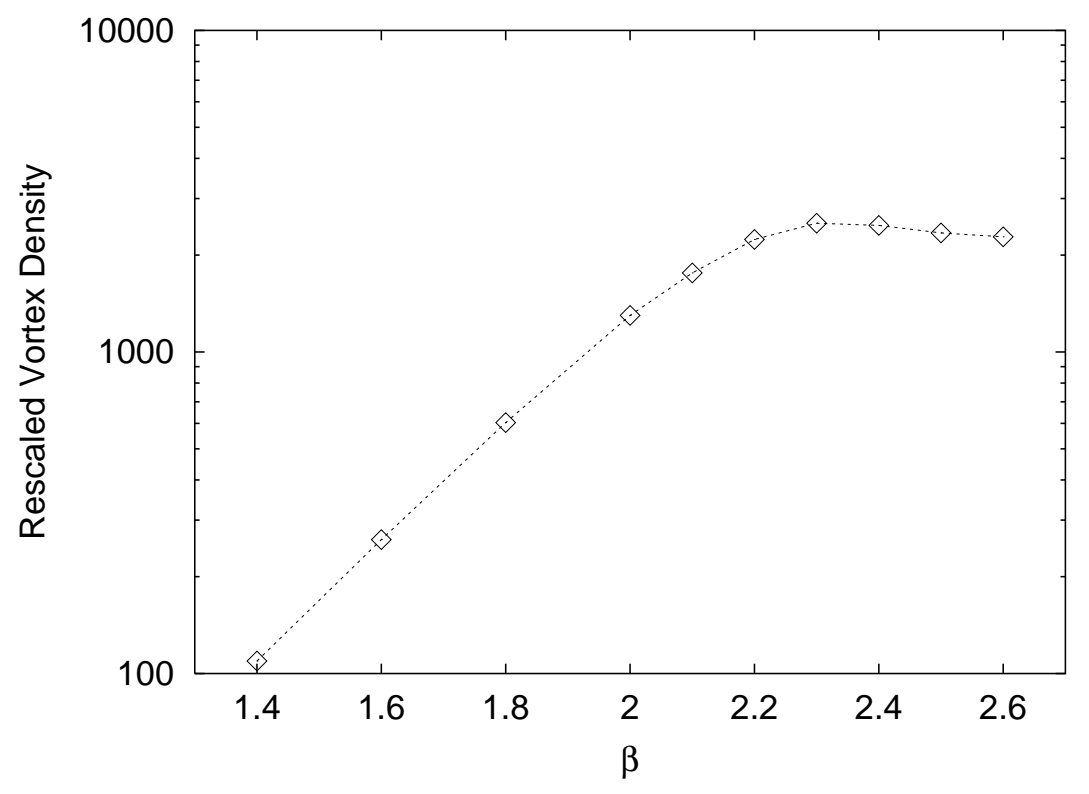

Figure 7: Rescaled vortex density $\tilde{p}=p / F(\beta)$, where $p$ is the measured vortex density and $F(\beta)$ is the asymptotic freedom expression in eq. (3.3). Vortex density scales if $\tilde{p}$ is constant.

\section{Vortex Thickness}

Why are such relatively large lattices required, in order to avoid large finite size effects in center projected Creutz ratios and vortex densities? We believe that the relevant length scale here is associated with the vortex thickness. Center vortices are surfacelike objects, in $D=4$ dimensions, which have a finite thickness in physical units. The thick vortex surface (or "core") bounds a Dirac 3-volume, which represents the region of discontinuity of a singular gauge transformation associated with the vortex. In ref. [17] we have explained the vortex-finding property of maximal center gauge in terms of the global properties of this singular gauge transformation. On a small 
lattice, however, with an extension comparable to the vortex thickness, these global aspects of the vortex field may be almost absent, and the minimal Dirac 3-volume could be quite small. In that case, the argument of ref. [17] breaks down. For this reason, we expect center projection to be less effective at finding vortices on small lattices, leading to underestimates of both the vortex density and the projected Creutz ratios.

Assuming there is some truth in this explanation, center projection can only be accurate for lattices whose extension is large compared to the vortex thickness. There are three independent ways of estimating the thickness of center vortices, which can be deduced from either

1. the ratio of "vortex-limited" Wilson loops [4];

2. the vortex free energy as a function of lattice size [14];

3. the adjoint string-breaking length [22].

Vortex-limited Wilson loops are defined in the following way: $W_{n}(C)$ is a Wilson loop evaluated on a sub-ensemble of unprojected configurations, selected so that precisely $n$ P-vortices, in the corresponding center-projected configurations, pierce the minimal area of the loop. We can further make the restriction, for $W_{1}(C)$, that the negative $\mathrm{P}$-vortex plaquette lies at (or touches) the center point of the rectangular loop. It is then expected that

$$
\frac{W_{1}(C)}{W_{0}(C)} \rightarrow-1
$$

in the limit where the vortex core is entirely contained within the loop (cf. ref. [4] for a more extended discussion). In Fig. 8 we show the data for $W_{1} / W_{0}$ vs. loop area at $\beta=2.3$, taken from our previous work in ref. [4]. Judging from this figure, the vortex appears to almost fit inside a $5 \times 5$ loop, which leads to a rough estimate of the vortex radius, as it pierces a plane, of about 3 lattice spacings. At $\beta=2.3$ we have $\sigma a^{2}=0.135$, and taking $\sigma=5 \mathrm{fm}^{-2}$, the lattice spacing is $a=0.164 \mathrm{fm}$. A diameter of 6 lattice spacings then corresponds to a vortex thickness of about one fermi.

A second estimate is obtained from the recent calculation of vortex free energy vs. lattice size, carried out numerically by Kovács and Tomboulis [14]. The vortex free energy is close to zero when the lattice extension is greater than the vortex thickness, and this again gives an estimate for the vortex thickness of a little over one fermi. Finally, if confinement is due to center vortices, then an $R \times T$ Wilson loop in the adjoint representation must change from a (Casimir scaling) area-law falloff to a (color-screening) perimeter-law falloff for charge separation $R$ greater than the vortex thickness [23]. The adjoint string-breaking distance has been measured, by de Forcrand and Philipsen [6], to be $1.25 \mathrm{fm}$, and this distance provides us with a third estimate of the vortex thickness, which is roughly consistent with the other two. 

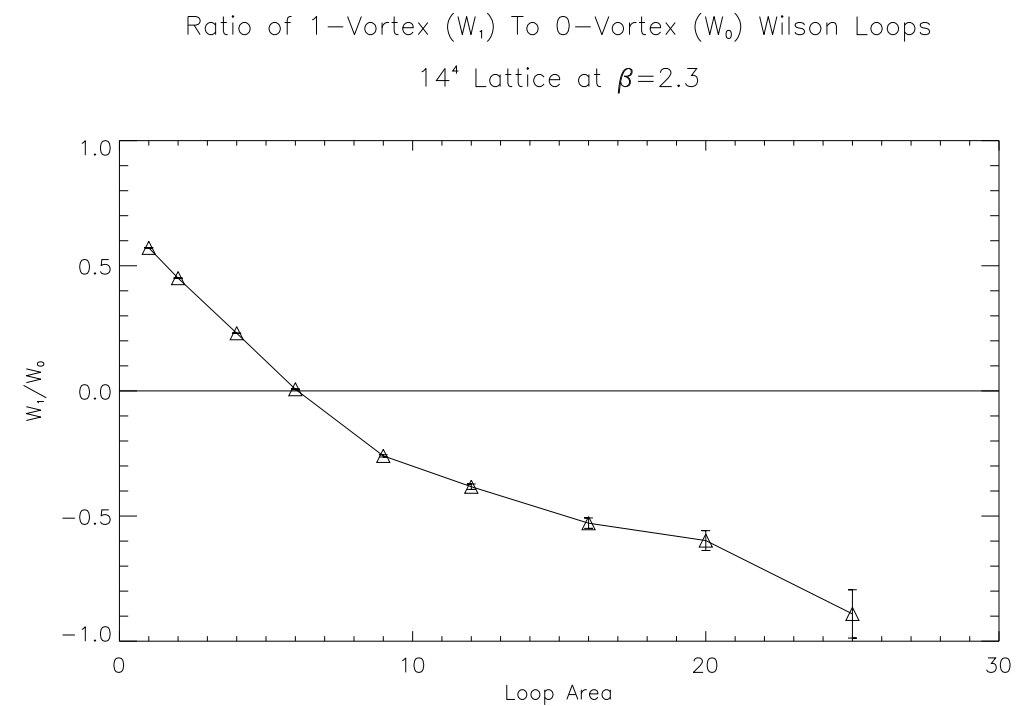

Figure 8: Ratio of the 1-Vortex to the 0-Vortex Wilson loops $W_{1}(C) / W_{0}(0)$, vs. loop area at $\beta=2.3$.

At $\beta=2.5$, one fermi corresponds to 12 lattice spacings. The lattice used by BKPV at $\beta=2.5$ was only 16 lattice spacings across, and this may simply be inadequate for center projection to reliably identify vortices, in view of the above estimates for the vortex thickness. In fact, the P-vortex density is quite low on small lattices, increasing sharply up to $L=16$ lattice spacings at $\beta=2.5$, where it begins to level off. This is illustrated in Fig. 9; the data points are the extrapolated values for $p$ at $N_{\text {copy }} \rightarrow \infty$.

In order to estimate the average distance between vortices, we need to know the vortex density. The P-vortex density, discussed in the previous section, is in fact an overestimate of the actual center vortex density $[10,12]$. The reason is that P-vortices fluctuate within the thick vortex core, and P-vortex locations, while correlated among random Gribov copies, do vary somewhat from one random copy to another [4]. Thus, although P-vortices are certainly near the middle of center vortices, they are unlikely to be exactly in the middle. A more accurate estimate of the center vortex density is arrived at by either "smoothing" the P-vortex surfaces, as explained in ref. [12], or else by taking the naive estimate of the number of vortices piercing a plane (per unit area in lattice units), extracted directly from the string tension (see, e.g., ref. [23])

$$
f=\frac{1}{2}\left(1-e^{-\sigma}\right)
$$

The two estimates agree fairly well [12], and for $\beta=2.3$ we find $f=0.063$. This implies an average distance of $f^{-1 / 2} \approx 4$ lattice spacings between the centers of vortex cores piercing a plane. Since we have already estimated the vortex thickness 


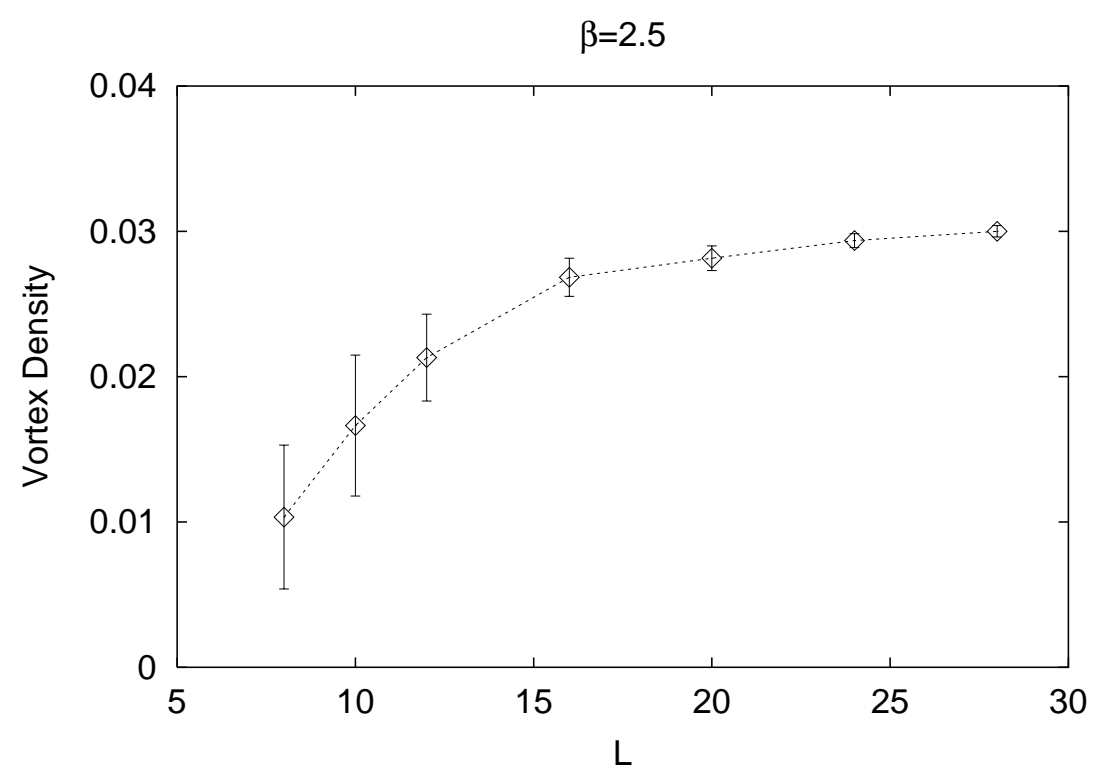

Figure 9: Vortex density $p$ vs. the extension of $L$ of the hypercubic lattice (volume $L^{4}$ ) at $\beta=2.5$.

at $\beta=2.3$ to be about 6 lattice spacings, its clear that there must be a substantial overlap between vortex cores, even on a very large lattice. There is nothing in principle wrong with that; vortex cores are not impenetrable objects, and their longrange effects are associated with Dirac 3-volumes, rather than the detailed structure of the core. Our findings for vortex thickness and separation simply indicate, in accordance with some old ideas of the Copenhagen group (Nielsen, Olesen, and Ambjørn in ref. [1]), that the QCD vacuum is more like a liquid of vortices than a dilute gas. Perhaps this is natural for objects which are both condensed and disordered.

\section{Conclusions}

We have found that center-projected lattices are more sensitive to finite size effects than are unprojected lattices, and precision results for center-projected Creutz ratios require lattice sizes which are large compared to one fermi. If accurate numbers (rather than just qualitative results) are required, then center-projected data from numerical simulations must be tested for convergence with respect to: (i) increasing the lattice size; (ii) increasing the number $N_{\text {copy }}$ of gauge copies used for center gauge fixing, and (iii) strengthening the gauge-fixing convergence criterion.

Our work was stimulated by the recent findings of BKPV [16], who have demonstrated a significant $N_{\text {copy }}$-dependence for center-projected Creutz ratios obtained on relatively small lattice volumes. In the large $N_{\text {copy }}$ limit, a large deviation $(\approx 30 \%)$ 
was found between the string tension on unprojected and projected lattices. Our result in the present article is that this $N_{\text {copy }}$ dependence is greatly reduced as lattice size increases, and center projected Creutz ratios $\chi_{c p}(I, I)$ appear to converge to values which are quite close to the asymptotic string tension obtained on unprojected lattices.

It is difficult to say whether the full and center-projected string tensions are in precise agreement in the infinite volume limit. To check such agreement, what is really required is an extrapolation to a triple limit: volume $\rightarrow \infty, N_{\text {copy }} \rightarrow \infty$, and $\delta \rightarrow 0$; our data is not yet adequate to extrapolate to this triple limit systematically. But we emphasize again that on the largest lattices we have used, and with results extrapolated (following BKPV) to the $N_{\text {copy }} \rightarrow \infty$ limit, the center projected Creutz ratios lie quite close to the asymptotic string tension, as illustrated in Fig. 1 above. Moreover, on the same large lattices and $N_{\text {copy }} \rightarrow \infty$ extrapolation, there is good evidence for asymptotic scaling of the vortex density at couplings $\beta>2.2$. The scaling of the vortex density, at the $\beta$ values studied, is in fact substantially better than the scaling of string tension itself.

Center projection in maximal center gauge is intended as a method for locating center vortices, on unprojected lattices, from the position of P-vortices on projected lattices. The correspondence of P-vortices with physical objects depends on

1. Scaling of the P-vortex density;

2. Correlation of P-vortex locations on the projected lattice with gauge-invariant observables on the unprojected lattice.

In particular, it is the correlation of P-vortices with unprojected Wilson loops, and specifically the ratios of "vortex-limited" Wilson loops $W_{n}(C) / W_{0}(C)$ on the unprojected lattice, which indicate that $\mathrm{P}$-vortices correspond to center vortices (rather than to some other type of object) on the unprojected lattice [5]. Moreover, if Pvortices locate center vortices, and if center vortices produce the full asymptotic string tension, then we must find

3. Center dominance; i.e. the equality of the string tension on projected and unprojected lattices.

The numerical simulations reported here were concerned with the scaling of the Pvortex density, and with center dominance. From the results of those simulations, we conclude that center projection in maximal center gauge remains a viable method of locating center vortices on full, unprojected lattices, and that these vortices probably account for the entire asymptotic string tension. 


\section{Acknowledgments}

Our research is supported in part by Fonds zur Förderung der Wissenschaftlichen Forschung P13997-PHY (M.F.), the U.S. Department of Energy under Grant No. DE-FG03-92ER40711 (J.G.), the "Action Austria-Slovakia: Cooperation in Science and Education" (Project No. 30s12) and the Slovak Grant Agency for Science, Grant No. 2/7119/2000 (S.O.). J.G. also acknowledges the support of MaPhySto, Centre

for Mathematical Physics and Stochastics, funded by the Danish National Research Foundation.

\section{References}

[1] G. 't Hooft, Nucl. Phys. B138 (1978) 1;

G. Mack, in Recent Developments in Gauge Theories, edited by G. 't Hooft et al. (Plenum, New York, 1980);

H. B. Nielsen and P. Olesen, Nucl. Phys. B160 (1979) 380;

J. Ambjørn and P. Olesen, Nucl. Phys. B170 (1980) 60; 265;

P. Vinciarelli, Phys. Lett. B78 (1978);

T. Yoneya, Nucl. Phys. B144 (1978) 195;

J. M. Cornwall, Nucl. Phys. B157 (1979) 392;

R. P. Feynman, Nucl. Phys. B188 (1981) 479.

[2] L. Del Debbio, M. Faber, J. Greensite, and Š. Olejník, Phys. Rev. D55 (1997) 2298, hep-lat/9610005.

[3] L. Del Debbio, M. Faber, J. Greensite, and Š. Olejník, in New Developments in Quantum Field Theory, ed. Poul Henrik Damgaard and Jerzy Jurkiewicz (Plenum Press, New York-London, 1998) 47, hep-lat/9708023.

[4] L. Del Debbio, M. Faber, J. Giedt, J. Greensite, and Š. Olejník, Phys. Rev. D58 (1998) 094501, hep-lat/9801027.

[5] M. Faber, J. Greensite, and Š. Olejník, JHEP 01 (1999) 008, hep-lat/9810008.

[6] Ph. de Forcrand and M. D’Elia, Phys. Rev. Lett. 82 (1999) 4582, hep-lat/9901020.

[7] C. Alexandrou, M. D'Elia, and Ph. de Forcrand, Nucl. Phys. Proc. Suppl. 83-84 (2000) 437, hep-lat/9907028; and Nucl. Phys. A663-664 (2000) 1031, hep-lat/9909005.

[8] K. Langfeld, H. Reinhardt, and O. Tennert, Phys. Lett. B419 (1998) 317, heplat/9710068.

[9] M. Engelhardt, K. Langfeld, H. Reinhardt, and O. Tennert, Phys. Rev. D61 (2000) 054504, hep-lat/9904004.

[10] M. Engelhardt, K. Langfeld, H. Reinhardt, and O. Tennert, Phys. Lett. B452 (1999) 301 , hep-lat/9805002. 
[11] J. Gattnar, K. Langfeld, A. Schäfke, and H. Reinhardt, hep-lat/0005016.

[12] R. Bertle, M. Faber, J. Greensite, and Š. Olejník, JHEP 03 (1999) 019, heplat/9903023.

[13] B. Bakker, A. Veselov, and M. Zubkov, Phys. Lett. B471 (1999) 214, hep-lat/9902010; M. Chernodub, M. Polikarpov, A. Veselov, and M. Zubkov, Nucl. Phys. Proc. Suppl. 73 (1999) 575, hep-lat/9809158.

[14] T. Kovács and E. Tomboulis, hep-lat/0002004;

E. Tomboulis, talk at Confinement 2000, Osaka, Japan.

[15] A. Hart, B. Lucini, Z. Schram, and M. Teper, JHEP 06 (2000) 040, hep-lat/0005010.

[16] V. Bornyakov, D. Komarov, M. Polikarpov, and A. Veselov, JETP Lett. 71 (2000) 231, hep-lat/0002017.

[17] M. Faber, J. Greensite, Š. Olejník, and D. Yamada, JHEP 12 (1999) 012, heplat/9910033.

[18] B. Bakker, A. Veselov, and M. Zubkov, hep-lat/0007022.

[19] K. Langfeld, talk at Quark Confinement and the Hadron Spectrum IV, Vienna, July $3-8,2000$.

[20] T. Kovács and E. Tomboulis, Phys. Lett. B463 (1999) 104, hep-lat/9905029.

[21] G. Bali, C. Schlichter, and K. Schilling, Phys. Rev. D51 (1995) 5165, hep-lat/9409005.

[22] P. de Forcrand and O. Philipsen, Phys. Lett. B475 (2000) 280, hep-lat/9912050.

[23] M. Faber, J. Greensite, and Š. Olejník, Phys. Rev. D57 (1998) 2603, hep-lat/9710039;

M. Faber, J. Greensite, and Š. Olejník, Acta. Phys. Slov. 49 (1999) 177, heplat/9807008. 